\title{
Pulsed Mode Radiofrequency Lesioning of the Suprascapular Nerve for the Treatment of Chronic Shoulder Pain
}

\author{
Rinoo V. Shah, MD, and Gabor B. Racz, MD
}

Suprascapular nerve blockade improves pain, range of motion, and disability in acute and chronic shoulder pain. Pain relief usually lasts several hours with local anesthetic. If steroids are added, the relief lasts several weeks. Since repetitive steroid exposure is associat-

Suprascapular nerve blockade plays an important role in managing acute, post-operative and chronic shoulder pain (1-5). Afferent nociceptive input from $70 \%$ of the shoulder joint will be blocked (1). Pain relief facilitates physical therapy and functional restoration of the shoulder (2). Pain relief typically lasts hours $(1,3,5)$, but can be prolonged if steroids are used $(2,4)$. Longer lasting procedures, such as neurolysis and neurectomy, are contraindicated: suprascapular neurolysis can lead to permanent paralysis of the supraspinatus and infraspinatus muscles. Pulsed mode radiofrequency (RF) lesioning is non-destructive but can relieve pain by delivering an electrical field to neural tissue (7). The mechanism is unknown. One theory suggests a neuromodulatory effect via changing gene expression in pain processing neurons (8). Anecdotal reports support its utility in long term pain control $(7,9)$.

\section{Case Report}

Two years prior to presentation, a 49-year old right handed man was involved in a motor vehicle collision. His

From Department of Anesthesiology and Pain Services, International Pain Institute, Texas Tech University Health Sciences Center, Lubbock Texas. Address Correspondence: Rinoo V. Shah, MD, International Pain Institute, Texas Tech University Health Sciences Center, 4430 South Loop 289, Lubbock, TX-79414. Email: rinoo_shah@yahoo.com

Funding: No external funding was received for this study and no financial benefit will be conferred on the authors nor on the device manufacturer mentioned herein. Dr. Gabor Racz acts as a consultant to Radionics ${ }^{\top \mathrm{M}}$, the manufacturer of the RFK ${ }^{\circledR}$ needle. ed with several hazards, alternative long-term therapies would be desirable. Pulsed mode radiofrequency is a non-destructive, safe, and repeatable long-term pain control therapy. We report a case, wherein a series of fluoroscopically guided, suprascapular nerve pulsed mode
RF treatments were performed. Each provided 4-5 months of pain relief and improvement in shoulder function, without deterioration in muscle strength.

Keywords: Radiofrequency, shoulder pain, suprascapular nerve block vehicle rolled over and he was ejected. He sustained multiple injuries including right glenoid, humeral, and clavicular fractures. Numerous shoulder surgeries were performed without success. He eventually developed severe, painful gleno-humeral osteoarthritis with adhesive capsulitis. The pain was present at night and aggravated by movement. Use of his arm was limited in all planes. He was disabled and unemployed. Despite physical therapy and oral analgesics, his pain averaged 7-8 out of 10 on the NRS-11 scale. Two separate orthopedic surgeons advised against a shoulder replacement or any other shoulder surgery. At presentation, active shoulder abduction measured 80 degrees and passive shoulder abduction measured 100 degrees. Active shoulder flexion measured 110 degrees and passive shoulder flexion measured 130 degrees. He was unable to place his right hand behind his neck. When reaching behind to his lower back, his right thumb only reached the lateral part of the lower buttock. Active internal and external rotation ranges of motion, with the arm at the side, were 20 and 30 degrees, respectively. There was tenderness to palpation over the coracoid process, delto-pectoral groove, and lateral acromial area. Crepitance was palpable and pain was reproduced with passive range of motion. Intra-articular glenohumeral joint injections were performed twice, but only provided relief for one week. In order to exclude a sympathetically maintained component to his pain, a stellate ganglion block was performed. This did not provide any relief. Inpatient hospital- ization was planned in order to place an interscalene, brachial plexus catheter. The infused concentration of local anesthetic and opioid would permit active physical therapy, while relieving pain. The insurance adjuster denied this, but approved a suprascapular nerve block. The block was performed under fluoroscopic guidance with 10 milliliters of a 1:1 mixture of $0.2 \%$ ropivacaine, $2 \%$ lidocaine, and $4 \mathrm{mg}$ of dexamethasone. There was a subjective, but substantial improvement in function and pain for three days. He requested a longer term solution.

Pulsed-mode RF of the suprascapular nerve was performed. A diagram of the scapula and the path of the suprascapular nerve is displayed (Fig. 1A). He was placed prone on the fluoroscopy table. The C-arm was obliqued about $16^{\circ}$ to the right and angled cephalo-caudad about $18^{\circ}$ (Fig. 1B). The suprascapular notch was easily identified superior to the scapular spine, medial to the coracoid process, and lateral to the rib margins (Fig. 2A). This image also demonstrates the fracture of the humeral head and the advanced glenohumeral osteoarthritis. After sterile preparation, a skin wheal with $1.5 \%$ lidocaine was raised overlying the notch. A 16-gauge Angiocath was advanced in a 'gun-barrel' fashion towards the notch. Then, a 20 gauge, $10 \mathrm{~cm}$ length, $10 \mathrm{~mm}$ active tip, curved blunt RFK ${ }^{\mathrm{TM}}$ (Radionics, Tyco Healthcare Group, 22 Terry Avenue, Burlington, MA, 01803) radiofrequency needle was advanced towards the notch (Fig. 2B). Sensory stimulation at 50 $\mathrm{Hz}, 0.2$ millisecond pulse width was per- 


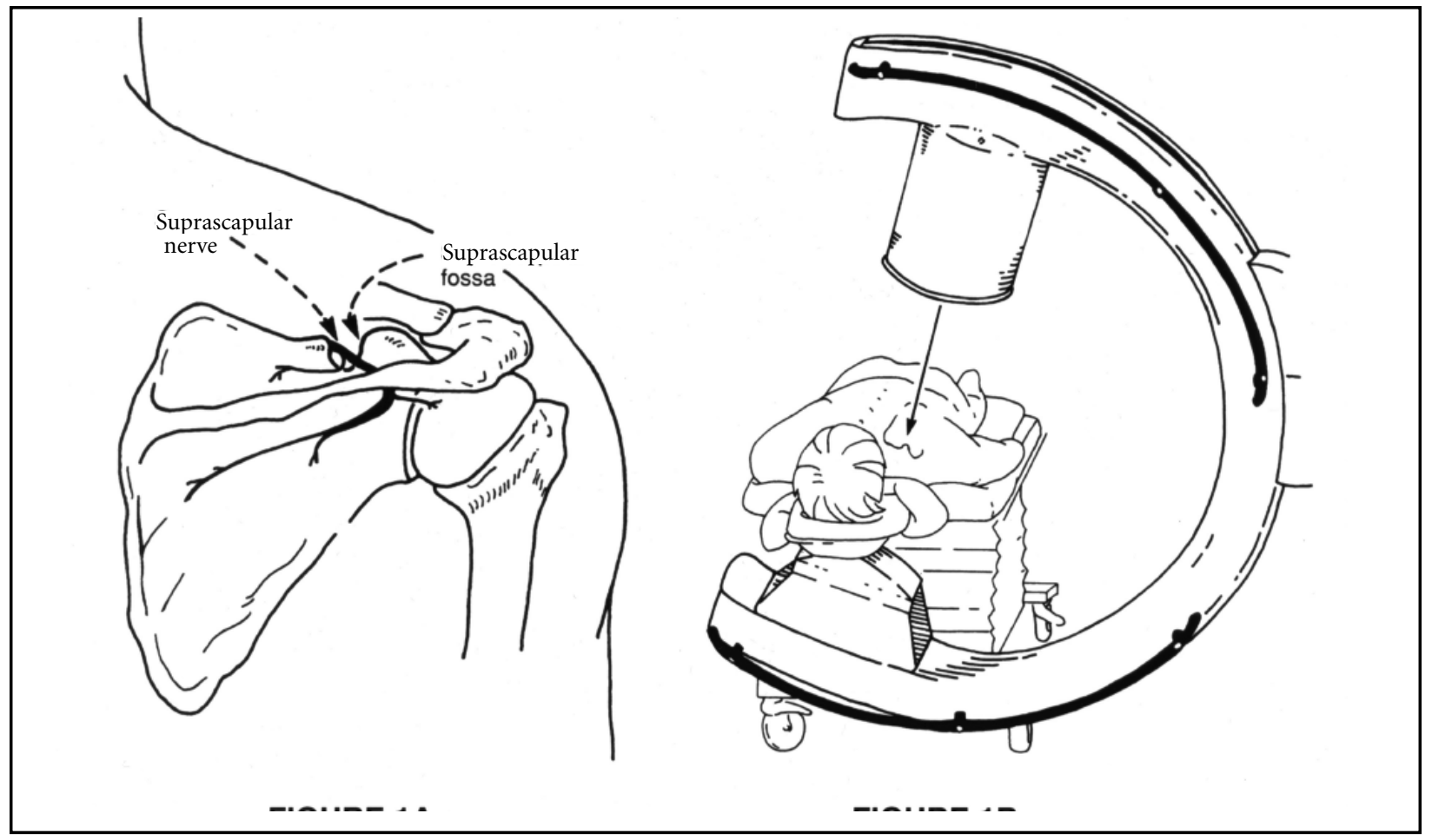

Fig 1A. Path of suprascapular nerve in relation to scapula and humerus B. Positioning of patient and C-arm for suprascapular nerve block. Reprinted with permission (22).

formed and reproducible paresthesias in the shoulder joint occurred at $0.3 \mathrm{~V}$. Motor stimulation at $2 \mathrm{~Hz}, 0.2$ millisecond pulse width was performed and contractions of the infraspinatus and supraspinatus muscles occurred at $0.4 \mathrm{~V}$. Pulsed mode RF lesioning was then carried out. The temperature was held constant at $42^{\circ} \mathrm{C}$. The electrical parameters used were a frequency of $2 \mathrm{~Hz}$ and a pulse width of

20 milliseconds. Three cycles of $120 \mathrm{sec}-$ onds each were performed. A local anesthetic mixture identical to the block, but without the steroid, was instilled at the end of the procedure. The pain intensity decreased to a 2-3. Active shoulder abduction improved to 110 degrees. Active shoulder flexion improved to 130 degrees. His thumb reached to the $1^{\text {st }}$ lumbar level. He, however, still had difficulty plac-

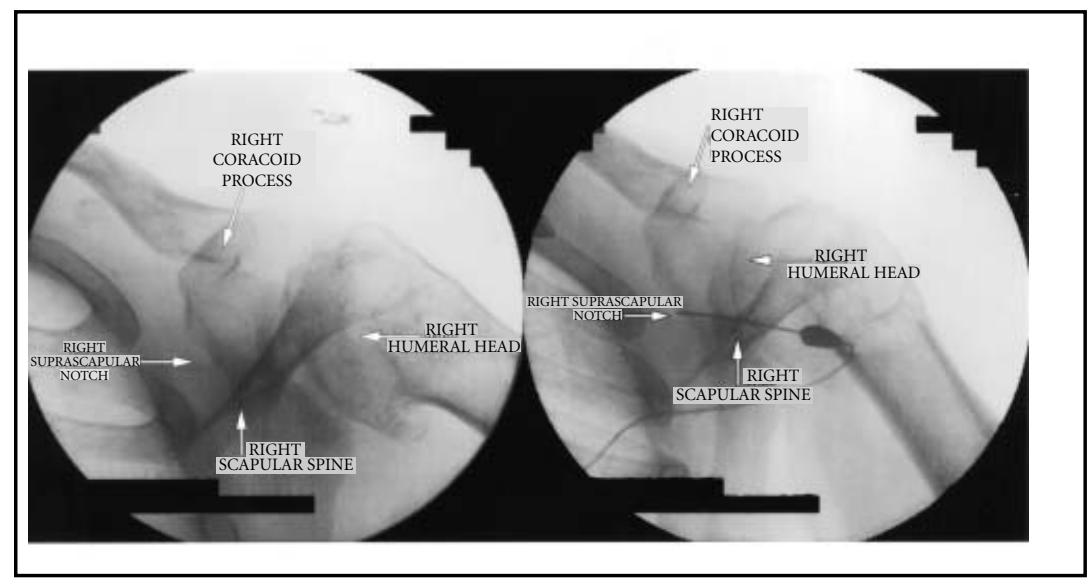

Fig. 2A. Fluoroscopic image of suprascapular notch and surrounding structures. B. RFK ${ }^{T M}$ pulsed RF needle in suprascapular notch ing his hand behind his neck. This improvement in pain and range of motion was sustained for 15 weeks but it gradually returned. The pulsed RF lesioning was repeated three more times. In total, 4 pulsed RF lesions were performed over 16 months. Average duration of pain relief varied from 12-18 weeks.

\section{Discussion}

The suprascapular nerve, derived from the C5 and C6 spinal nerves, leaves the upper trunk of the brachial plexus. It runs inferiorly, posteriorly, and laterally to reach the scapula. Upon passing underneath the transverse scapular ligament and through the suprascapular notch, the suprascapular nerve enters the supraspinous fossa. The nerve terminates as articular and muscular branches (1). Subscapular, axillary, and lateral pectoral nerve branches innervate the anterior shoulder joint capsule, but the suprascapular nerve provides the main innervation to the posterior shoulder joint capsule, acromioclavicular joint, subacromial bursa, and coracoclavicular ligament (10). The suprascapular nerve also supplies motor and sensory innervation, particularly noci- 
ception and proprioception, to the infraspinatus and supraspinatus muscles (11). Overall, the suprascapular nerve provides sensory innervation to approximately $70 \%$ of the shoulder girdle (1).

Chronic shoulder pain is typically thought of as a structural or musculoskeletal problem. However, the peripheral nerves innervating the shoulder joint may have been damaged or sensitized at the time of initial injury or subsequent surgery (10). Chronic shoulder pain may be better classified in terms of inflammatory and neuropathic processes (2). Regional neural blockade treats both types of pain by blocking nociceptive transduction and transmission (1). The prompt relief provided by regional blockade and the distribution of nerves to the shoulder girdle suggest that selective neural blockade can diagnose and treat refractory shoulder problems (10).

Suprascapular nerve blockade alone has been useful for post-operative pain control following arthroscopic shoulder surgery (3). Even when combined with blockade of the brachial plexus, suprascapular nerve blockade can prolong post-operative analgesia by $58 \%$ in nonarthroscopic shoulder surgery (5). Suprascapular nerve blockade has been helpful in treating chronic shoulder pain secondary to adhesive capsulitis, gleno-humeral osteoarthritis, rheumatoid arthritis, and chronic rotator cuff injuries (1, 3, 12-14). The shoulder range of motion may also improve $(1,2,14)$. These clinical studies support the neuroanatomic hypothesis that the suprascapular nerve is an important mediator of shoulder pain $(1,3,5$, 10). Unfortunately, the effects of suprascapular nerve blockade are short-lived and thus, limit its usefulness in the long term $(1,2,5,14)$. Even two studies with the longest follow-up of 12 weeks demonstrated either no improvement or a gradual deterioration in the range of motion with time $(2,14)$. Improvement in active range of motion, over placebo, occurred primarily with abduction and flexion, but not external rotation or placement of hand behind the back $(2,14)$. Despite these small gains and short-lived effects, one author advocated these blocks as a bridge to surgical intervention (14). Nonetheless, repetitive steroid exposure through nerve blocks is associated with several side effects and complications (6). Alternative long-term solutions would be useful.
Peripheral neurolysis has been used to provide long term relief of refractory neuropathic pain that is responsive to a diagnostic block $(15,16)$. Dellon et al (16), demonstrated that peripheral neurolysis of the terminal branches of the saphenous nerve can treat refractory knee pain. Neurolysis of the suprascapular nerve has rarely been reported $(17,18)$. Lewis et al (16), demonstrated improvements in pain and range of motion in patients suffering from shoulder arthridities. Mallon et al (17) described neurolysis of the suprascapular nerve for the treatment of suprascapular neuropathy. However, in their two cases the nerves were actually decompressed rather than neurolysed (17).

Several methods of neurolysis are available: surgical, chemical, and RF thermocoagulation (6). RF neurolysis is unique among these methods: the lesion volume is controlled and the morbidity is low (6). In conventional RF neurolysis, a high frequency electrical current is delivered through an insulated needle. Due to surrounding tissue impedance, local molecular vibrations are induced. As energy is dissipated, heat is generated (6) Temperatures typically reach $60-80$ degrees Celsius and cause tissue denaturation (6). Irreversible damage to neural tissues, however, occurs above 45 degrees Celsius $(19,20)$. Irrespective of the neurolytic method, irreversible nerve damage is associated with several major problems: permanent loss of function, deafferentation pain, neuroma formation, and neuritis (6). True neurolysis of mixed nerves is a contraindication in non-cancer pain (9).

Recent evidence suggests that the electrical field, rather than the heat lesion, is responsible for the clinical effect of RF $(19,20)$. Pulsed RF is a non-neurolytic lesioning method that provides relief of experimental and clinical neuropathic pain (6-8). A placebo controlled, double blind study demonstrated that pulsed electrical fields relieve pain and improve function in osteoarthritis (21). Short pulses of radiofrequency energy, delivered at a constant temperature, produce central and peripheral neuromodulatory effects (8). The precise mechanisms of pain relief are unknown, but may involve alterations in the expression of genes such as c-fos (8). Temperatures in pulsed RF, unlike conventional RF, typically do not exceed 42 degrees C: the time between puls- es is sufficient to allow dissipation of heat and thus, lower temperatures. The risks of neuritis, deafferentation pain, and neuroma formation are minimal. Even if identical temperatures are used, pulsed RF demonstrates better efficacy than conventional RF (19). Pulsed RF may provide long lasting pain relief, reduction in analgesic use, and patient satisfaction (7). Furthermore, the procedure can be repeated if the pain recurs, since no tissue has been destroyed. Pulsed RF of the suprascapular nerve may benefit patients with chronic shoulder pain and disability (16).

Suprascapular nerve blockade is typically performed with the use of anatomic landmarks and Euclidean geometry (1, $2,5)$. The scapula is divided into 4 quadrants by the intersection of two lines. One line is drawn along the scapular spine and another bisects the inferior scapular pole. The upper outer quadrant is further bisected and the needle is inserted 1.5 to $2.5 \mathrm{~cm}$ radially along this line $(1,2,5)$. Despite fluoroscopic validation (2), this technique lacks precision and ultimately, successful blockade will depend on the spread of local anesthetic $(2,14)$. Greater precision is obtained by the use of a nerve stimulator: elicitation of paresthesias in the posterior shoulder joint and contractions of the infraspinatus and supraspinatus are essential $(3,5,16)$. However, pneumothorax is still a rare possibility with a blind technique (5). Fluoroscopy (2) and electrical stimulation (1) may be combined to optimize both precision localization and safety (22). This method is straightforward, reproducible, and safe. We have used this method, over the past two years, in more than three hundred shoulders. There have been no complications.

Chronic shoulder pain secondary to glenohumeral osteoarthritis presents a challenge. Our patient may have been a candidate for a hemiarthroplasty or total shoulder arthroplasty. In patients under the age of 50, pain may be alleviated with these procedures (23). However, when a rating scale was applied, more than half had an unsatisfactory result (23). Hemiarthroplasties for glenohumeral OA required revision more often than rheumatoid arthritis (23). The authors (23) advised pursuing alternative treatment strategies. Pain management, in this population, typically consists of analgesics and physical therapy. Intraarticular injections in our patient provided only 
one week of relief. Suprascapular nerve blockade proved to be a useful diagnostic and therapeutic tool. Pulsed RF lesioning of the suprascapular nerve, using fluoroscopy and electrical stimulation, provided 12-18 weeks of subjective pain relief and functional improvement. Even when the pain recurred, the technique was easily repeated. Clinicians should be aware of this novel approach to the treatment of intractable shoulder pain, when all else fails.

\section{Conclusion}

Pulsed RF lesioning of the suprascapular nerve may improve pain control and function in patients suffering from intractable shoulder pain. Fluoroscopy and electrical stimulation complement one another in enhancing the safety and efficiency of this technique.

Author Affiliation:
Rinoo V. Shah, MD
Assistant Professor
International Pain Institute
Texas Tech University Health Sciences
Center
4430 South Loop 289
Lubbock, TX 79414
E-mail: rinoo_shah@yahoo.com
Gabor B. Racz, MD
Grover E. Murray Professor and
Chairman Emeritus
International Pain Institute
Texas Tech University Health Sciences
Center
$36014^{\text {th }}$ Street
Lubbock, TX 79430
E-mail: paula.brasher@ttuhsc.edu

\section{REFERENCES}

1. Karatas GK, Meray J. Suprascapular nerve block for pain relief in adhesive capsulitis: comparison of 2 different techniques. Arch Phys Med Rehabil 2002; 83:593-597.

2. Shanahan EM, Ahern M, Smith $M$ et al. Suprascapular nerve block (using bupivacaine and methylprednisolone acetate) in chronic shoulder pain. Ann Rheum Diseases 2003; 62:400-406.

3. Ritchie ED, Tong D, Chung F et al. Suprascapular nerve block for postoperative pain relief in arthroscopic shoulder surgery: A new modality? Anesth Analg 1997; 84:1306-1312.

4. Jones DS, Chattopadhyay C. Suprascapular nerve block for the treatment of frozen shoulder in primary care: A randomized trial. Br J Gen Pract 1999; 49:39-41.

5. Neal J, McDonald S, Larkin K et al. Suprascapular nerve block prolongs analgesia after nonarthroscopic shoulder surgery but odes not improve outcome. Anesth Analg 2003; 96: 982-986.

6. Shah RV, Ericksen JJ, Lacerte M. Interven tions in chronic pain management. 2. New frontiers: Invasive nonsurgical interventions. Arch Phys Med Rehabil 2003; 84: S39-S44.

7. Munglani R. The longer term effect of pulsed radiofrequency for neuropathic pain. Pain 1999; 80:437-439.

8. Higuchi $Y$, Nashold BS Jr., Sluijter $M$ et al. Exposure of the dorsal root ganglion in rats to pulsed radiofrequency currents activates dorsal horn lamina I and II neurons. Neurosurgery 2002; 50:850-856.

9. Rohof OJ. Pulsed radiofrequency of the suprascapular nerve in the treatment of chronic intractable shoulder pain. In Raj PP (ed). $2^{\text {nd }}$ World Congress of W.I.P. Istanbul: Blackwell Science; 2001:114.

10. Aszmann OC, Dellon AL, Birely BT et al. Innervation of the human shoulder joint and its implications for surgery. Clin Orthop 1996; 330:202-207.

11. Minaki Y, Yamashita T, Takebayashi T et al. Mechanosensitive afferent units in the shoulder and adjacent tissues. Clin Or thop 1999; 369:349-356.

12. Brown DE, James DC, Roy S. Pain relief by suprascapular nerve block in gleno-humeral arthritis. Scand / Rheumatol 1988; 17:411-415.

13. Emery P, Bowman S, Wedderburn L et al. Suprascapular nerve block for chronic shoulder pain in rheumatoid arthritis. BMJ 1989; 299:1079-1080.

14. Vecchio PC, Adebajo AO, Hazleman BL. Suprascapular nerve block for persistent rotator cuff lesions. / Rheumatol 1993; 20: 453-455.

15. Dellon A, Mont M, Mullick T et al. Partial denervation of persistent neuroma pain around the knee. Clin Orthop 1996; 329: 216-222.

16. Rohof O. Radiofrequency treatment of peripheral nerves. Pain Practice 2002; 2: 257-260.

17. Lewis R. The use of combined suprascapular and circumflex (articular branches) nerve blocks in the management of chronic arthritis of the shoulder joint. Eur / Anaesthesiol 1999; 16:37-41.

18. Mallon W, Bronec P, Spinner R et al. Suprascapular neuropathy after distal clavicle excision. Clin Orthop 1996; 329:207-211.

19. Sluijter M, Racz G. Technical aspects of radiofrequency. Pain Practice 2002; 2:195-200.

20. Van Zundert, J., Raj P, Erdine $S$ et al. Application of radiofrequency treatment in practical pain management: State of the art. Pain Practice 2002; 2:269-278.

21. Trock D, Bollet A, Dyer $R$ et al. A double blind trial of the clinical effects of pulsed electromagnetic fields in osteoarthritis. Rheumatology 1993; 20:456-460.

22. Raj PP. Suprascapular nerve block. In Raj PP, Lou L, Erdine S et al (eds). Radiographic Imaging for Regional Anesthesia and Pain Management. $1^{\text {st }}$ ed. Churchill Livingstone; Philadelphia, 2003, pp 128-131.

23. Sperling J, Cofield R, Rowland C. Neer hemiarthroplasty and Neer total shoulder arthroplasty in patients fifty years old or less. Long term results. JBJS 1998; 80:464-473. 